\title{
The untapped world of reaction gases with regards to LA-ICP-MS/MS
}

\section{MR. THOMAS ZACK}

University of Gothenburg

Presenting Author: thomas.zack@gu.se

Especially in the field of LA-ICP-MS, setups that utilize the wide range of possible ion-molecule reactions have seen an exponential increase of application in recent years. This is primarily due to the introduction of commercially available systems that enable to quantify the products of ions reacting with gas molecules within a designated area of an ICP-MS called reaction cells. Ion-molecule reactions can be extremely selective, so that even monisotopic interferences are separated (e.g., separating ${ }^{87} \mathrm{Sr}^{+}$from ${ }^{87} \mathrm{Rb}^{+}$by forming ${ }^{87} \mathrm{SrO}^{+}$molecules while reacting with $\mathrm{N}_{2} \mathrm{O}$; here ${ }^{87} \mathrm{Rb}^{+}$is not reacting). (please also see: http://www.yorku.ca/dkbohme/research/selection_table.html).

In particular, beta-decay based geochronometers have become part of the geochemist's toolbox by utilizing such selective reactivity from a wide variety of reaction gases (e.g., using $\mathrm{N}_{2} \mathrm{O}$ and $\mathrm{SF}_{6}$ for $\mathrm{Rb}-\mathrm{Sr}$ dating, $\mathrm{NH}_{3}$ for $\mathrm{Lu}-\mathrm{Hf}$ dating, and $\mathrm{CH}_{4}$ for $\mathrm{Re}-$ Os dating). One aspect that has been given less attention to is the fact that such reaction gases are not only removing unwanted interferences in specialized applications, but can rather be an integral part of routinely determining element concentrations during LA-ICP-MS. For example, during in-situ Rb-Sr dating of micas by using $\mathrm{N}_{2} \mathrm{O}$ as a reaction gas, concentration data for more than 30 major and trace elements are collecting routinely in Gothenburg. This requires a solid knowledge of all possible reaction products for those elements.

In this contribution, I will outline a simple routine how an overview can be gathered on ion-molecule reactions for more than 70 elements. Specific reaction behavior is critically dependent on instrumental setup (e.g., the kinetic energy of an ion colliding with a gas molecule can vary by several orders of magnitude), but can still be extremely useful in a given setup.

A surprisingly selective behavior of ion-molecule reactions was observed during such investigations. This ultimately leads to a complete reevaluation of best strategies for quantifying LAICP-MS measurements using the best possible peak to background ratios and least risk of interferences. Furthermore, the advent of combining reaction cells and MC-ICP-MS opens up several new isotope systems (e.g., Ti isotope systematics) with new challenges of removing interferences. 